\title{
PLASMA EDGE MODIFICATION IN STRONGLY COMPENSATED SEMICONDUCTORS
}

\author{
W. Szuszkiewicz, K. DYвко \\ Institute of Physics, Polish Academy of Sciences \\ Al. Lotników 32/46, 02-668 Warszawa, Poland \\ W. BARDYSZEWSKI \\ Institute of Theoretical Physics, Warsaw University \\ Hoża 69, 00-681 Warszawa, Poland \\ AND C. JULIEN \\ Laboratoire de Physique des Solides, Université Pierre et Marie Curie \\ 4, pl. Jussieu, 75252 Paris, Cedex 05, France
}

We demonstrate that the electron-impurity interaction can modify the reflectivity in the vicinity of plasma minimum giving rise to a small dip on the plasma edge. Experimental spectra taken for $\mathrm{Hg}_{1-x} \mathrm{Co}_{x} \mathrm{Se}$ for $x<0.02$ at various temperatures confirm this theoretical prediction. The position of the structure can be used to determine the plasma frequency in highly compensated materials at low temperatures.

PACS numbers: $71.45 . \mathrm{Gm}, 78.30 . \mathrm{Fs}, 72.30 .+\mathrm{q}$

The infrared absorption coefficient is most often described in terms of the simple Drude-Zener theory assuming frequency independent damping constants of free-carrier plasma and phonon excitations. Such approximation can be used to interpret the experimental data taken for lightly doped or uncompensated materials. However, in heavily doped and/or highly compensated semiconductors some deviations from such "classical" picture can be expected due to the increasing role of the collective plasma (plasmon) excitations in the process of the free-carrier absorption. Plasmon generation in the process of photon absorption by free-electron gas in semiconductors is possible only if ionized impurities or other imperfections breaking the translational invariance are present in the system. Resonant behaviour of the free-carrier loss function above the plasma frequency leads to the enhancement of the damping parameter in the ac conductivity.

A lot of studies have been devoted to the role of collective excitations in the free-carrier absorption. They were primarily related to the question of plasmon excitation in the process of electron-impurity scattering (for the references see, 
e.g. [1]). It was also known that in heavily doped materials electron plasma modifies dynamic properties of the crystalline lattice leading to the renormalization of the longitudinal optical phonon oscillations [2]. The effect of dynamical screening of electron-impurity interaction by phonons has been analyzed in Ref. [3]. Usually, in order to determine the absorption coefficient experimentally, both transmission and reflection measurements have to be performed. However, precise determination of the transmission coefficient is particularly difficult in the vicinity of the plasma edge. On the other hand, for typical II-VI or III-V compounds the plasmon generation related structure in absorption is not directly observable on the reflection curves. One can see the trace of the plasmon generation process due to carrier-impurity scattering only by using a deconvolution procedure by which the absorption coefficient is calculated from the transmission and reflection spectra. However, as the rate of plasmon generation in the free-carrier absorption critically depends on the concentration of the Coulomb scattering centers, one can expect more pronounced effects in highly compensated materials.

In this paper we would like to consider the situation where the plasma frequency is much higher than LO phonon frequency so that we can concentrate on the plasmon-like branch of the collective excitation spectra. Such conditions are relatively easy to achieve in narrow-gap and zero-gap semiconductors. In this case it is convenient to characterize the dominant, electron-impurity scattering rate by the frequency dependent inverse relaxation time given by the formula

$$
1 / \tau(\omega)=\frac{\omega^{2} m^{*}}{e^{2} n} \operatorname{Re} \sigma(\omega)
$$

where $n$ and $m^{*}$ denote free electron concentration and effective mass, respectively. The real part of the high frequency conductivity $\sigma(\omega)$ is calculated to the lowest order in the electron-impurity interaction in the current-conserving approximation $[4,5]$

$$
\begin{array}{r}
\operatorname{Re} \sigma(\omega)=-\frac{2 e^{4} N_{\mathrm{imp}}}{\omega^{3} 3 \pi m^{* 2}} \int_{0}^{+\infty} \mathrm{d} q q^{2}\left(\frac{\epsilon(q, 0)}{f+\epsilon(q, 0)}\right)^{2} \operatorname{Im}\left(\frac{1}{\epsilon(q, \omega)}\right) \\
\times \frac{\left[\omega^{2}-\omega_{\mathrm{TO}}^{2}(1+f / \epsilon(q, 0))\right]^{2}}{\left\{\omega^{2}-\omega_{\text {TO }}^{2}[1+\operatorname{Re}(f / \epsilon(q, \omega))]\right\}^{2}+\left[\omega_{\mathrm{TO}}^{2} \operatorname{Im}(f / \epsilon(q, \omega))\right]^{2}},
\end{array}
$$

where $\epsilon(q, \omega)$ denotes the dielectric function without the lattice contribution, $f=$ $\epsilon_{0}-\epsilon_{\infty}$ is the difference between the static and high frequency dielectric constant and $\omega_{\mathrm{TO}}$ is the transverse optical phonon frequency.

The electron-impurity scattering rate is proportional to the loss function $\operatorname{Im}(-1 / \epsilon(q, \omega))$ multiplied by a resonant term which is important only in the spectral range close to the optical phonon band frequencies [3]. Number of ionized impurities $N_{\text {imp }}=D n$ depends on the compensation ratio

$$
D=\frac{N_{\mathrm{D}}+N_{\mathrm{A}}}{N_{\mathrm{D}}-N_{\mathrm{A}}}
$$

where $N_{\mathrm{D}}$ and $N_{\mathrm{A}}$ are the concentrations of ionized donors and ionized acceptors, respectively.

With the increasing $D$ value (the increasing number of Coulomb centers) one can expect the increase in the electron-impurity scattering rate. As a consequence 
the slight modification of the optical constants (in particular - of the reflectivity) should be seen in the vicinity of the free-carrier plasma frequency. The effect should be especially strong at the concentration of impurity centers much higher than the electron concentration (under the condition of strong compensation).

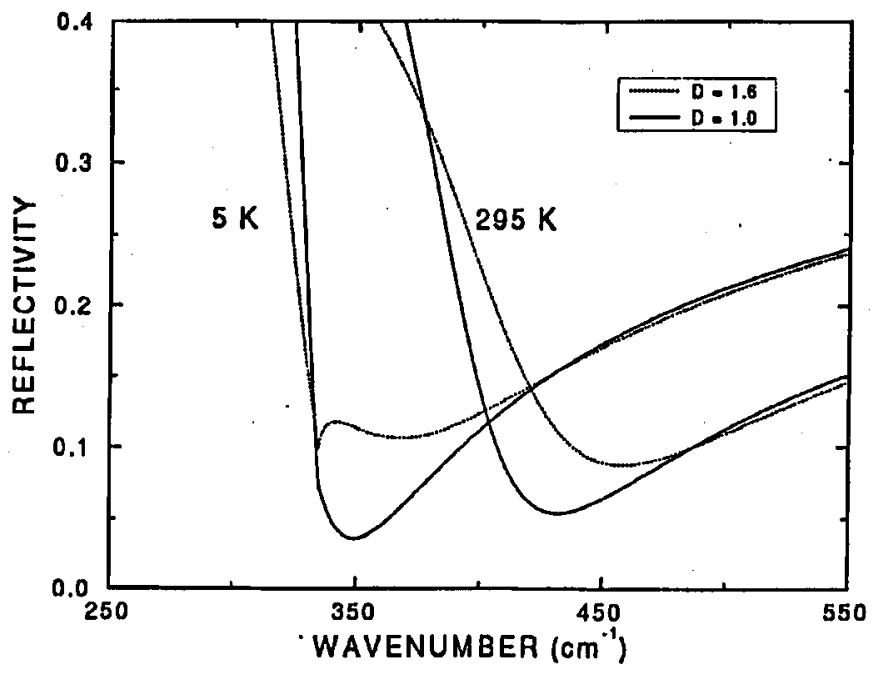

Fig. 1. Theoretical dependence of the reflectivity on the frequency $\omega$ in the vicinity of the plasma frequency calculated for parameters corresponding to one of $\mathrm{HgSe}$ samples doped with Co. The effect of the increasing compensation ratio on the reflectivity at $5 \mathrm{~K}$ and $295 \mathrm{~K}$ is demonstrated.

The calculated dependence of the reflectivity on the frequency $\omega$ in the vicinity of plasma minimum is presented in Fig. 1. Both the influence of the temperature and of the compensation ratio are shown in this figure. Small but well pronounced minimum at the exact position of plasma frequency at $T=5 \mathrm{~K}$ results from the modification of the impurity scattering. The amplitude of the structure is sensitive to both temperature and doping level, being the highest at low temperature and high compensation. The expected shape of the reflectivity curve modification should enable one to make experimental observation of the effect.

In order to verify the theoretical predictions the infrared reflectivity spectra have been taken on the transition metal doped IgSe samples. Two kinds of crystals have been selected for the optical investigations: $\mathrm{HgSe}$ doped with $\mathrm{Fe}$ and $\mathrm{HgSe}$ doped with Co. As it is well known Fe in HgSe is a resonant donor [6] and the compensation ratio $D$ should be close to 1 for this material. To the contrary, Co impurity in $\mathrm{HgSe}$ acts as an acceptor (at least for the high doping concentration range) $[7,8]$ and a nonvanishing compensation can be easily obtained in this case.

Single crystals of $\mathrm{Hg}_{1-x} \mathrm{Fe}_{x} \mathrm{Se}$ and $\mathrm{Hg}_{1-x} \mathrm{Co}_{x} \mathrm{Se}$ for $x<0.02$ were grown by a modified Bridgman method. The Fe or Co concentration for the samples used in the optical experiments has been determined by the electron microprobe and the energy-dispersive X-ray fluorescence (EDXRF) analysis. The surface of 
the samples has been prepared by the chemical etching in $\mathrm{Br} /$ methanol solution. The reflectivity has been measured using the Fourier spectrometer in the spectral range from $200 \mathrm{~cm}^{-1}$ to $1000 \mathrm{~cm}^{-1}$ with the typical instrument resolution equal to $1 \mathrm{~cm}^{-1}$. One millimeter thick samples were mounted on the cold finger of the helium cryostat.

A reasonable agreement between the Drude model and the experimental reflectivity spectrum can be achieved at room temperature for all samples by treating the free-carrier plasma frequency and damping as fitting parameters. However, the experimental data obtained for $\mathrm{Hg}_{1-x} \mathrm{Co}_{x} \mathrm{Se}$ at low temperatures revealed a new feature close to the expected position of the plasma frequency. In Fig. 2 the typical experimental reflectivity spectra taken in the discussed frequency range are presented. There is no step-like structure at room temperature, but the well-pronounced structure can be seen at $T=5 \mathrm{~K}$. A certain modification of the plasma edge can be observed in a wide temperature range (even to about $200 \mathrm{~K}$ in some cases). The width of the structure is correlated with the plasmon frequency band width. Such effect does not exist for $\mathrm{IIg}_{1-x} \mathrm{Fe}_{x}$ Se samples.

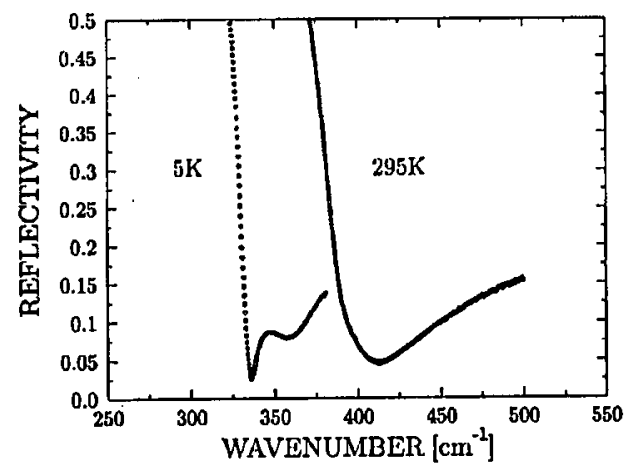

Fig. 2. Selected experimental reflectivity spectra taken for one of $\mathrm{Hg}_{0.995} \mathrm{Co}_{0.005} \mathrm{Se}$ samples using a Fourier spectrometer (instrument resolution equal to $0.5 \mathrm{~cm}^{-1}$ ).

In conclusion we would like to state that the scattering of electrons on ionized impurities in ionic crystals can lead to the strong modification of the optical constants at low temperatures. In particular this effect is manifested by a sharp dip in the reflectivity spectra at the position of the free-carrier plasma frequency. According to the theoretical model the feature in the reflectivity increases with the increasing compensation ratio. High resolution reflectivity measurements can thus provide direct information about the plasma frequency $\omega_{\mathrm{pl}}$ in highly compensated semiconductor at low temperatures.

We would like to express our gratitude to Mrs. B. Witkowska for growing the samples used for our investigations.

\section{References}

[1] W. Bardyszewski, W. Szuszkiewicz, Acta Phys. Pol. A 77, 273 (1990). 
[2] M.E. Kim, A. Das, S.D. Senturia, Phys. Rev. B 18, 6890 (1978).

[3] W. Szuszkiewicz, W. Bardyszewski, C. Julien, Acta Phys. Pol. A 87, 555 (1995).

[4] S. Goettig, J. Phys. C, Solid State Phys. 17, 4443 (1984).

[5] W. Bardyszewski, W. Szuszkiewicz, Qian Dingrong, Zhang Jiaming, J. Cryst. Growth 101, 792 (1990).

[6] J. Kossut, W. Dobrowolski, in: Handbook of Magnetic Materials, Ed. K.H. Buschow, Vol. 7, Elsevier Science Publishers B.V., Amsterdam 1993, Chap. 4, pp. 231-305.

[7] W. Szuszkiewicz, E. Dynowska, S. Miotkowska, B. Witkowska, C. Julien, M. Balkanski, Nukleonika 39, 99 (1994).

[8] W. Szuszkiewicz, M. Arciszewska, B. Witkowska, C. Julien, M. Balkanski, J. Magn. Magn. Mater. 140-144, 2037 (1995). 